Proceedings of the International Congress on Advances in Applied Physics and Materials Science, Antalya 2011

\title{
Coupled Multiphysics Models for the Analysis of the Conduction and Valence Band Eigenenergies in Cylindrical Quantum Dots
}

\author{
S. Prabhakar, E. Takhtamirov and R. Melnik* \\ ${ }^{b} \mathrm{M}^{2} \mathrm{NeT}$ Laboratory, Wilfrid Laurier University, 75 University Avenue West, Waterloo, ON, N2L 3C5, Canada
}

\begin{abstract}
We develop a mathematical model for the analysis of conduction and valence band eigenenergy in quantum dots. We apply the model to study the band structure calculations of low dimensional semiconductor nanostructures such as wurtzite AlN/GaN quantum dots in cylindrical coordinates in presence of applied magnetic field along $z$-direction. We use a finite element method to solve the resulting model and to obtain eigenvalues and wave functions of cylindrical quantum dots. We provide details of the methodology of solution and appropriate boundary conditions. A special attention is given to the case of applied magnetic field along $z$-direction, we found localized eigenstates and wave functions in the conduction and valence bands for which our results open new possibilities for the design of the optoelectronics devices where the combination of electron-hole pairs can be used as tuning parameters.
\end{abstract}

PACS: 71.15.--m, 73.21.La, 62.23.Eg

\section{Introduction}

Nanostructures based on wide band gap semiconductor materials such as AlN/GaN quantum dots have attracted significant attention due to their current and potential applications in optical, optoelectronic and electronic devices, as well as bionanotechnology. The small, arbitrary shaped crystal structures can be realized to obtain quantum size effects patterning techniques with the help of electron beam lithography, optical lithography, X-ray lithography and others. Self-assembled QDs which are grown by the Stranski-Krastanov process are of special interest because of their potential applications in QDs lasers, light emitting diodes as qubits for quantum computation and other applications [1-4].

A subject that has not been studied in detail in the context of AlN/GaN quantum dots, however, is related to localized eigenstates in valence band of wurtzite structures in cylindrical coordinates and this is the subject of the present investigation. Our approach is closely related to that of [1] but differs in a way that we include the external magnetic field along $z$-direction and derive the equations for holes in the valence band in cylindrical coordinates with a different procedure.

In this paper, we also present a numerical analysis of the band structure of single AlN/GaN QDs under the influence of applied magnetic field along $z$-direction. By using the finite element method (FEM), we study the eigenvalues and wave functions of the electrons in the conduction and valence bands.

\section{Mathematical model}

* corresponding author; e-mail: rmelnik@wlu.ca
In this section, we develop a theoretical model of the Hamiltonian, focusing on both the conduction and valence band in cylindrical coordinates of quantum wurtzite system. The detailed theoretical model for the conduction band of wurtzite structures in cylindrical coordinates was explained in [4]. The results on the valence band Hamiltonian in cylindrical coordinates will be published elsewhere [5].

\subsection{Conduction band Hamiltonian in cylindrical coordinates}

We consider the motion of the electron confined along the $z$-direction in the presence of magnetic field oriented along a direction perpendicular to the plane of two-dimensional electron gas (2DEG). Therefore the total Hamiltonian of an electron including Rashba spin orbit interaction in cylindrical coordinates can be written as [4]:

$$
H=\left[\begin{array}{cc}
h_{+} & \alpha_{\mathrm{R}}\left[\frac{\partial}{\partial r}+\frac{1}{r}\left(j+\frac{1}{2}\right)+\frac{e B r}{2 \hbar}\right] \\
\alpha_{\mathrm{R}}\left[-\frac{\partial}{\partial r}+\frac{1}{r}\left(j-\frac{1}{2}\right)+\frac{e B r}{2 \hbar}\right] & h_{-}
\end{array}\right],
$$

where

$$
\begin{aligned}
h_{ \pm} & =-\frac{\hbar^{2}}{2 m_{0}}\left[\frac{1}{r} \frac{\partial}{\partial r} \frac{r}{m_{\mathrm{e}}^{\perp}} \frac{\partial}{\partial r}+\frac{\partial}{\partial z} \frac{1}{m_{\mathrm{e}}^{\|}} \frac{\partial}{\partial z}\right. \\
& \left.-\frac{1}{r^{2}} \frac{1}{m_{\mathrm{e}}^{\perp}}\left(j \mp \frac{1}{2}\right)^{2}\right]+\frac{1}{8} m_{\mathrm{e}}^{\perp} \omega_{\mathrm{c}}^{2} \rho^{2}+\frac{\hbar \omega_{\mathrm{c}}}{2}\left(j \mp \frac{1}{2}\right) \\
& \mp \frac{1}{2} g_{0} \mu_{\mathrm{B}} B+E_{\mathrm{c}},
\end{aligned}
$$

where $\omega_{\mathrm{c}}=e B / m_{\mathrm{e}}^{\perp} c$ is the cyclotron frequency, $m_{\mathrm{e}}^{\perp}$ and $m_{\mathrm{e}}^{\|}$ are the effective masses, $\mathrm{c}$ is the velocity of light and $B$ is the magnetic field along $z$-direction. Also, $\alpha_{\mathrm{R}}$ is the Rashba coefficient. Here we consider the experimentally reported bulk material parameters $g_{0}=1.9885$ for AlN 
and $g_{0}=1.9510$ for GaN material from Ref. [6].

\subsection{Valence band Hamiltonian in Cartesian coordinates}

We start from the steady state Schrödinger equation in the effective-mass approximation [7],

$$
H F=\left(H^{0}+H^{\text {so }}+H^{k}+H^{\varepsilon}\right) F=E F .
$$

Equation (3) was constructed by using the orthogonal and normalized Bloch zone-center functions for the potential well material, which are written as $\boldsymbol{u}=\left(u_{x}, u_{y}, u_{z}\right)$. The functions $u_{x}=u_{x}(\boldsymbol{r})$ and $u_{y}=u_{y}(\boldsymbol{r})$ transform as the Cartesian coordinates $x$ and $y$ belonging to the representation $\Gamma_{6}$ of the space group $C_{6 v}$, and $u_{z}=u_{z}(\boldsymbol{r})$ transforms as the coordinate $z$ along $c$-axis of the wurtzite structure in $\Gamma_{1}$ notation [8]. The envelope function $F=F(\boldsymbol{r})$ has three components: $F=\left(F_{x}, F_{y}, F_{z}\right)^{\mathrm{T}}$, where $\mathrm{T}$ stands for

the transpose of a vector or operator, so that total wave function $\Psi=\Psi(\boldsymbol{r})$ can be written as

$$
\Psi=\sum_{j=x, y, z} u_{j} F_{j}=\boldsymbol{u} \boldsymbol{F} .
$$

The basis functions $\boldsymbol{u}$ are spinless, so each envelope function element $F_{j}$ act as a spinor. The Hamiltonian $H^{0}, H^{\text {so }}$ and $H^{k}$ in Eq. (3) can be written as [8]:

$$
\begin{aligned}
H^{0} & =\left(\begin{array}{ccc}
U_{6} & 0 & 0 \\
0 & U_{6} & 0 \\
0 & 0 & U_{1}
\end{array}\right), \\
H^{\mathrm{so}} & =\mathrm{i}\left(\begin{array}{ccc}
0 & -\Delta_{2} \sigma_{z} & \Delta_{3} \sigma_{y} \\
\Delta_{2} \sigma_{z} & 0 & -\Delta_{3} \sigma_{x} \\
-\Delta_{3} \sigma_{y} & \Delta_{3} \sigma_{x} & 0
\end{array}\right),
\end{aligned}
$$

$$
H^{k}=\left(\begin{array}{ccc}
L_{1} k_{x}^{2}+M_{1} k_{y}^{2}+M_{2} k_{z}^{2} & N_{1} k_{x} k_{y} & N_{2} k_{x} k_{z} \\
N_{1} k_{x} k_{y} & M_{1} k_{x}^{2}+L_{1} k_{y}^{2}+M_{2} k_{z}^{2} & N_{2} k_{y} k_{z} \\
N_{2} k_{x} k_{z} & N_{2} k_{y} k_{z} & M_{3}\left(k_{x}^{2}+k_{y}^{2}\right)+L_{2} k_{z}^{2}
\end{array}\right),
$$

where $U_{6}=U(\boldsymbol{r})$ and $U_{1}=U_{1}(\boldsymbol{r})$ are the edges of the bands $\Gamma_{6}$ and $\Gamma_{1}$, respectively, which may contain an external scalar potential. $\sigma_{i}$ are the Puali matrices. $L_{1}, L_{2}, M_{1}, M_{2}, N_{1}$ and $N_{2}$ are the material parameters for the potential well semiconductor, and $\hbar k$ is the momentum operator. In Eq. (7), we choose the relation $L_{1}-M_{1}=N_{1}$.

\subsubsection{Valence band Hamiltonian in cylindrical coordinates}

All Cartesian elements from Eq. (3) can be changed into cylindrical coordinates $L_{1}-M_{1}=N_{1}(r, \phi, z)$ by allowing $x=r \cos \phi$ and $y=r \sin \phi$. Here we suppose the envelope functions $\overline{\bar{F}}=\mathrm{e}^{\mathrm{i} m \phi} f(r, z) / \sqrt{2 \pi}$. Here the eigenvalues $m$ of the operator of the $z$-component of the total angular momentum should be half-integer: $m= \pm 1 / 2, \pm 3 / 2, \ldots$ The detailed procedure for the conversion of the Hamiltonian in cylindrical coordinates will be published elsewhere [5]. Here we present the main result. The Hamiltonian in cylindrical coordinates can be written as

$$
\overline{\bar{H}}^{k}=-\left(\begin{array}{ccc}
h_{11} & \mathrm{i} L_{1} \nabla_{r} \frac{m}{r}-\mathrm{i} M_{1}\left[\frac{m}{r} \nabla_{r}+\frac{m}{r^{2}}\right] & N_{2} \nabla_{r} \nabla_{z} \\
\mathrm{i} L_{1}\left[\frac{m}{r} \nabla_{r}+\frac{m}{r^{2}}\right]-\mathrm{i} M_{1} \nabla_{r} \frac{m}{r} & h_{22} & \mathrm{i} N_{2} \frac{m}{r} \nabla_{z} \\
N_{2}\left[\nabla_{r}+\frac{1}{r}\right] \nabla_{z} & \mathrm{i} N_{2} \frac{m}{r} \nabla_{z} & h_{33}
\end{array}\right),
$$

where

$$
\begin{aligned}
& h_{11}=L_{1}\left(\nabla_{r}^{2}+\nabla_{r} \frac{1}{r}\right)-M_{1} \frac{m^{2}}{r^{2}}+M_{2} \nabla_{z}^{2}, \\
& h_{22}=M_{1}\left(\nabla_{r}^{2}+\nabla_{r} \frac{1}{r}\right)-L_{1} \frac{m^{2}}{r^{2}}+M_{2} \nabla_{z}^{2}, \\
& h_{33}=M_{3}\left(\nabla_{r}^{2}+\frac{1}{r} \nabla_{r}-\frac{m^{2}}{r^{2}}\right)+L_{2} \nabla_{z}^{2},
\end{aligned}
$$

and the matrix $\boldsymbol{m}$ has the simple form

$$
\boldsymbol{m}=\left(\begin{array}{cc}
m-\frac{1}{2} & 0 \\
0 & m+\frac{1}{2}
\end{array}\right) .
$$

The spin-orbit interaction Hamiltonian (6) transforms into the Hamiltonian $\tilde{H}^{\text {so }}=S H^{\text {so }} S^{-1}$ :

$$
\tilde{H}^{\text {so }}=\mathrm{i}\left(\begin{array}{ccc}
0 & -\Delta_{2} \sigma_{z} & \Delta_{3} \sigma_{\phi} \\
-\Delta_{2} \sigma_{z} & 0 & -\Delta_{3} \sigma_{r} \\
-\Delta_{2} \sigma_{\phi} & \Delta_{2} \sigma_{r} & 0
\end{array}\right),
$$

where $\sigma_{r}=\sigma_{x} \exp \left(\mathrm{i} \phi \sigma_{z}\right)$ and $\sigma_{\phi}=\sigma_{y} \exp \left(\mathrm{i} \phi \sigma_{z}\right)$.

The Hamiltonian (13) depends on $\phi$ and does not commute with the operator $-\mathrm{i} \nabla_{\phi}$. To eliminate this dependence, we note first that

$$
\mathrm{e}^{\mathrm{i} \frac{\phi}{2} \sigma_{z}} \sigma_{r} \mathrm{e}^{-\mathrm{i} \frac{\phi}{2} \sigma_{z}}=\sigma_{x}, \quad \mathrm{e}^{\mathrm{i} \frac{\phi}{2} \sigma_{z}} \sigma_{\phi} \mathrm{e}^{-\mathrm{i} \frac{\phi}{2} \sigma_{z}}=\sigma_{x},
$$

and then we construct a unitary transformation $\tilde{S} \tilde{F}=\tilde{\tilde{F}}$, where

$$
\tilde{S}=\left(\begin{array}{ccc}
\mathrm{e}^{\mathrm{i} \frac{\phi}{2} \sigma_{z}} & 0 & 0 \\
0 & \mathrm{e}^{\mathrm{i} \frac{\phi}{2} \sigma_{z}} & 0 \\
0 & 0 & \mathrm{e}^{\mathrm{i} \frac{\phi}{2} \sigma_{z}}
\end{array}\right) .
$$




\subsubsection{Inclusion of external magnetic field}

Consider an external magnetic field $B_{z}$ applied along the $z$-axis, which does not break the cylindrical symmetry of the system. We use the symmetric gauge for the vector potential $\boldsymbol{A}=\left(A_{x}, A_{y}, A_{z}\right)=(-y B / 2,-x B / 2,0)$ in cylindrical coordinates, or $\boldsymbol{A}=\left(A_{r}, A_{\phi}, A_{z}\right)=\left(0, r B_{z} / 2,0\right)$ in cylindrical coordinates. The kinetic momentum operator is $K=k+e A /(\hbar c)$, where $e$ is the electronic charge. Also, we have to include the effective Pauli term in the Hamiltonian of (3) as

$$
H^{B}=\left(\begin{array}{ccc}
\frac{1}{2} g_{0} \mu_{\mathrm{B}} B \sigma_{z} & -\frac{\mathrm{i} e}{2 \hbar c} Q B & 0 \\
0 & \frac{1}{2} g_{0} \mu_{\mathrm{B}} B \sigma_{z} & 0 \\
\frac{\mathrm{i} e}{2 \hbar c} Q B & 0 & \frac{1}{2} g_{0} \mu_{\mathrm{B}} B \sigma_{z}
\end{array}\right) \text {, }
$$

where $g_{0}=2$ is the free electron $g$-factor, $\mu_{\mathrm{B}}$ is the Bohr magneton, and $Q$ is a material specific parameter [9]. In a similar way that was discussed in the previous section, in the presence of external magnetic field along $z$-direction, one can write the Hamiltonian in cylindrical coordinates as

$$
\overline{\bar{H}}^{k}=-\left(\begin{array}{ccc}
h_{11} & h_{12} & N_{2} \nabla_{r} \nabla_{z} \\
h_{12} & h_{22} & \mathrm{i} N_{2} \frac{m}{r} \nabla_{z} \\
N_{2}\left(\nabla_{r}+\frac{1}{r}\right) \nabla_{z} & \mathrm{i} N_{2} \frac{m}{r} \nabla_{z} & h_{33}
\end{array}\right),
$$

where $h_{11}, h_{22}$ and $h_{33}$ are given by (9)-(11) and

$$
\begin{aligned}
& h_{12}=\mathrm{i} L_{1}\left(m, \nabla_{r}\right)_{+} \frac{1}{2 r}-\mathrm{i} M_{1}\left[\frac{1}{2 r}\left(m, \nabla_{r}\right)+\frac{m}{r^{2}}\right], \\
& h_{21}=\mathrm{i} L_{1}\left[\frac{1}{2 r}\left(m, \nabla_{r}\right)_{+}+\frac{m}{r^{2}}\right]-\mathrm{i} M_{1}\left(m, \nabla_{r}\right)_{+} \frac{1}{2 r},
\end{aligned}
$$

and the matrix $\boldsymbol{m}$ has the simple form

$$
\boldsymbol{m}=\left(\begin{array}{cc}
\frac{e}{2 \hbar c} B_{z} r^{2}+m-\frac{1}{2} & 0 \\
0 & \frac{e}{2 \hbar c} B_{z} r^{2}+m+\frac{1}{2}
\end{array}\right) .
$$

\section{Results and discussions}

We consider the GaN as a quantum dot embedded into the host material of AlN. We apply the Dirichlet boundary conditions far away from the quantum dot. In other words, the electron wave function is zero sufficiently far away from the quantum dot. Further, we consider the electron wave function to be continuous at the center and at the interface between AlN/GaN quantum dot and we apply Neumann boundary condition at the center and at the interface of the AlN/GaN quantum dot. We found the localized eigenstates and eigenvalues in the conduction and valence bands of wurtzite AlN/GaN quantum dot. The electron and hole eigenstates found in the conduction and valence bands recombine each other in the form of electron-hole pairs and produce photons. These photons have wide area of applications in light emitting diodes, semiconductor lasers, and others.

In Fig. 1, we plotted the conduction and valence band diagram of AlN/GaN quantum dot along z-direction by choosing the appropriate band discontinuity and band offset at the interface of the $\mathrm{AlN} / \mathrm{GaN}$ heterojunction. We adopted the finite element method to solve the coupled Schrödinger Eq. (1) for an electron in cylindrical

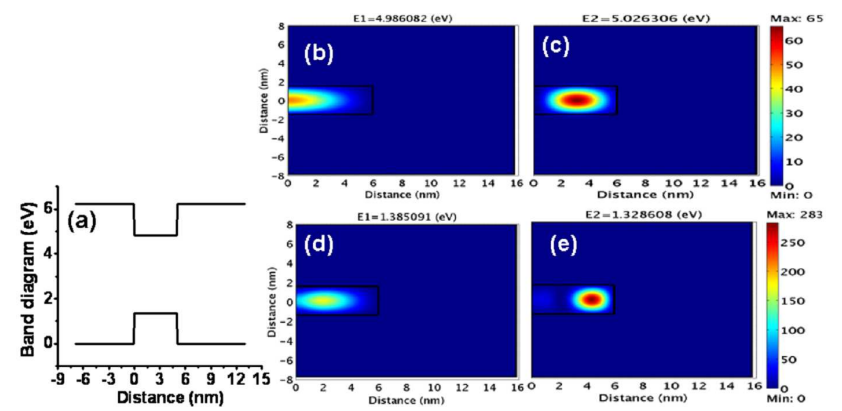

Fig. 1. (a) Flat conduction and valence band diagram of AlN/GaN quantum dot along z-direction, (b) ground state eigenvalues and wave functions of electron in the flat conduction band, (c) first excited state eigenvalues and wave functions of electron in the flat conduction band, (d) ground state eigenvalues and wave functions of holes in the flat valence band, (e) first excited state eigenvalues and wave functions of holes in the flat valence band.

coordinates in the flat conduction band. The eigenvalues and the wave functions of the ground and first excited states of the electron are shown in Fig. 1a and b. Here we estimated the ground and first excited states eigenvalues in the conduction band as $4.986082 \mathrm{eV}$ and $5.026306 \mathrm{eV}$, respectively. The ground and first excited states of the electron in the valence band have been found by solving the corresponding eigenvalue problem for the Hamiltonian (1). The ground and first excited states of the electron wave functions are shown in Fig. 1d and e. Here, we estimated the ground and first excited states eigenvalues of the electron as $1.385091 \mathrm{eV}$ and $1.328608 \mathrm{eV}$. The electron found in the localized eigenstates at $4.986082 \mathrm{eV}$ in the conduction band has an opportunity to recombine with the hole found in the localized eigenstates at $1.385091 \mathrm{eV}$ in the valence band. The recombination of electron-hole pair generates photons which are fundamental to the operation of many optoelectronic semiconductor devices, such as photodiodes, light emitting diodes (LEDs) and laser diode.

\section{Conclusions}

In this paper, we have developed a mathematical model for the valence band of a quantum dot in cylindrical coordinates with wurtzite symmetry in the presence of external magnetic field along $z$-direction. We solved the coupled Schrödinger equation in the conduction and valence bands separately and found the localized eigenstates and wave functions. The localized eigenstates for electron and hole in their corresponding bands will recombine to form electon-hole pairs. The photons generated by electronhole pairs during the recombination process has wide applications for the optoelectronic devices. 


\section{Acknowledgments}

This work was supported by the NSERC and the CRC program, Canada.

\section{References}

[1] L.C. Lew Yan Voon, C. Galeriu, B. Lassen, M. Willatzen, R. Melnik, Appl. Phys. Lett. 87, 041906 (2005).

[2] B. Daudin, F. Widmann, G. Feuillet, Y. Samson, J.L. Rouviere, Phys. Rev. B 56, R7069 (1997); P. Micheler, Single Quantum Dots, Fundamentals, Applications and New Concepts, Springer, Berlin 2003.

[3] S.R. Patil, R. Melnik, Nanotechnology 20, 125402 (2009).
[4] S. Prabhakar, R. Melnik, J. Appl. Phys. 108, 064330 (2010).

[5] S. Prabhakar, E. Takhtamirov, R. Melnik, J. Appl. Phys., in preparation.

[6] M.W. Bayerl, M.S. Brandt, T. Graf, O. Ambacher, J.A. Majewski, M. Stutzmann, Phys. Rev. B 63, 165204 (2010).

[7] J.M. Luttinger, W. Kohn, Phys. Rev. 97, 869 (1955).

[8] S.L. Chuang, C.S. Chang, Phys. Rev. B 54, 2491 (1996).

[9] J.M. Luttinger, Phys. Rev. 102, 1030 (1956). 